\title{
Los manglares de Costa Rica: el Pacífico norte
}

\author{
Priscilla Zamora-Trejos ${ }^{1 \dagger}$ \& Jorge Cortés ${ }^{1,2}$ \\ 1. Centro de Investigación en Ciencias del Mar y Limnología (CIMAR), Ciudad de la Investigación, Universidad de \\ Costa Rica, 11501-2060 San Pedro, San José, Costa Rica; jorge.cortes@ucr.ac.cr \\ 2. Escuela de Biología, Universidad de Costa Rica, 11501-2060 San Pedro, San José, Costa Rica.
}

Recibido 22-IX-2008. Corregido 24-II-2009. Aceptado 25-III-2009.

\begin{abstract}
Costa Rica mangroves: the north Pacific. Costa Rica has mangrove forests on both the Caribbean and Pacific coasts. The Pacific side has $99 \%$ of the mangrove area of the country. In this review we compile available information on the mangroves of the north Pacific coast of Costa Rica, from Bahía Salinas, on the border with Nicaragua, to the tip of the Peninsula de Nicoya at Cabo Blanco. We provide information on the location of the mangroves and all available information for each mangrove forest. These mangrove communities are smaller in extension and tree sizes, and have lower diversity compared to the mangroves on the southern section of the Pacific coast of Costa Rica. The dominant species are Rhizophora mangle and Rhizophora racemosa along the canal edges, backed by Avicennia germinans, and farther inland Avicennia bicolor, Laguncularia racemosa and Conocarpus erectus. At Potrero Grande a healthy population of Pelliciera rhizophorae, a rare species, has been reported. We recognized 38 mangrove communities in this part of the country, based on the National Wetland Inventory, published papers, field observations, theses, technical reports, and the national topographic maps (1:50 000, Instituto Geográfico Nacional). Relatively detailed information could be found for only five mangrove forests, for 14 more only prelimary and incomplete lists of plants and in some cases of animal species are available, for nine there is even less information, and for nine more only their location is known, which in some cases was not correct. Detail mapping, characterization of the vegetation and fauna, physiological studies, analyses of biogeochemical and physical processes, economic valuations, and determination of the health status of the mangrove of the northern Pacific coast, as well as for the rest of Costa Rica, are neccesary and urgent. Rev. Biol. Trop. 57 (3): 473-488. Epub 2009 September 30.
\end{abstract}

Key words: Mangroves, Costa Rica, eastern tropical Pacific, human impact, Guanacaste.

Los manglares son uno de los ecosistemas más amenazados del mundo. $\mathrm{Su}$ existencia se encuentra aún en más peligro que la de los bosques tropicales y los arrecifes de coral (Valiela et al. 2001, Duke et al. 2007). La situación particular para Costa Rica no es distinta a la de otros manglares en el mundo, pues las principales actividades que amenazan a estos ecosistemas también son practicadas en el país. La tala para producción de madera, carbón y taninos se practicó en el pasado, y en algunas zonas costeras aún se realiza a nivel artesanal (Solórzano et al. 1991, Pizarro \& Angulo 1993, Bilio et al. 1999). La proliferación de salineras y estanques para camarones es otra causa importante de deterioro de los manglares del planeta (Solórzano et al. 1991, Jiménez 2004), y en Costa Rica esta podría ser la causa principal de la reducción de los manglares del Pacífico Norte y Pacífico Central. El cambio del uso de la tierra, para construcción de puertos o la tala del bosque para convertirlo en pastizales y zonas agrícolas, o para hoteles y marinas, están causando daños irreversibles en estos ecosistemas costeros, entre otras cosas, debido al degradación en la calidad del agua (Jiménez 2004). Se ha indicado que entre 1964-1989 hubo una reducción de un 27\% de la cobertura de manglar en el Pacífico de Costa Rica (MIRENEM 1993). 
La información existente sobre los manglares de Costa Rica se generó principalmente durante la década de 1980 cuando hubo una proliferación de investigadores que estudiaban diversos temas sobre los manglares costarricenses (e.g. Castaing et al. 1980, Villalobos et al. 1985, Jiménez \& Soto 1985, Soto \& Corrales 1987, Jiménez 1988). Posterior a esta época y hasta la actualidad se han generado diversas publicaciones, algunas de ellas dirigidas hacia el manejo y la explotación adecuada de los recursos (e.g. Cruz 1999, Silva-Benavides \& Bonilla 2001, Germain 2004, Silva \& Carillo 2004). Adicionalmente, muchos de los estudios se han concentrado en ciertas regiones: Punta Morales, Tivives y Térraba-Sierpe. Los manglares en Costa Rica cubren solamente el 0.8\% del territorio nacional, pero su importancia biológica y económica es muy grande. Se extienden a lo largo de todo la costa Pacífica, desde Bahía Salinas hasta Golfo Dulce, con el 99\% de los manglares ubicados en esta costa (Pizarro \& Angulo 1993, Polanía 1993). El resto de los manglares se ubican en la parte sur de la costa Caribe, principalmente representados por el manglar de Gandoca-Manzanillo, el más extenso de esa región (Coll et al. 2001).

Sistematizar la información que existe sobre los manglares de Costa Rica no solo permitirá conocer cuál es el estado del conocimiento de los mismos, sino también identificar zonas en las que se han dado cambios a través del tiempo y definir elementos de estudio para el desarrollo futuro de investigaciones en estas zonas tan vulnerables y de gran importancia biológica, social y económica. En el presente trabajo se describen los manglares ubicados en el Pacífico norte de Costa Rica, desde Bahía Salinas hasta la Reserva Absoluta de Cabo Blanco, en la punta sur de la Península de Nicoya. Se incluye toda la información encontrada sobre sus características físicas, químicas y biológicas.

\section{MATERIALES Y MÉTODOS}

Entre el 2002 y el 2005 se recopiló y analizó toda la bibliografía existente sobre los manglares de Costa Rica. Se revisaron bases de datos en línea y se visitaron diferentes bibliotecas del país. Se incluyeron libros, artículos científicos, documentos mimeografiados, tesis e informes técnicos. Además, se consultaron diversos mapas de humedales que se han elaborado. También se consultó a expertos en el tema sobre referencias que debían ser incluidas. Adicionalmente, se hicieron visitas de campo para corroborar algunos de los datos mencionados.

La información obtenida fue analizada y resumida por sitio, presentando las características físico-químicas, tipo de vegetación y especies estudiadas. Adicionalmente, se elaboraron mapas donde se muestra cada uno de los manglares y se mencionan los manglares que hasta el momento no han sido descritos o mencionados en la literatura.

Los manglares estudiados en el presente trabajo se ubican desde Bahía Salinas hasta Cabo Blanco (Fig. 1). Todos estos manglares pertenecen a la región climática denominada como Pacífico Norte. Esta región se caracteriza por poseer una estación seca prolongada y una precipitación anual de 1500 a $2000 \mathrm{~mm}$ (Jiménez \& Soto 1985). En el inventario de humedales de Costa Rica se indican 38 manglares en esta región (Córdoba-Muñoz et al. 1998) (Fig. 1, Cuadro 1). Dentro del Sistema de Áreas de Conservación de Costa Rica estos manglares se ubican en el Área de Conservación Guanacaste (ACG) y Área de Conservación Tempisque (ACT).

\section{DESCRIPCIÓN DE LOS MANGLARES}

\section{Área de Conservación Guanacaste}

\section{Manglar de Puerto Soley}

Ubicación: Se ubica en Bahía Salinas, Guanacaste, aproximadamente en los $11^{\circ} 03$ '32”N (11 $04^{\prime}$ 'según Córdoba-Muñoz et al. 1998; 11³6’N según Tejada-Rivas 2002) y $85^{\circ} 40^{\prime} \mathrm{W}$ (Fig. 2). Este comprende un área de 200 hectáreas (Jiménez 1994) establecido sobre una planicie costera de sustrato arenoso y está directamente conectado con el mar (Soto \& Jiménez 1982). 


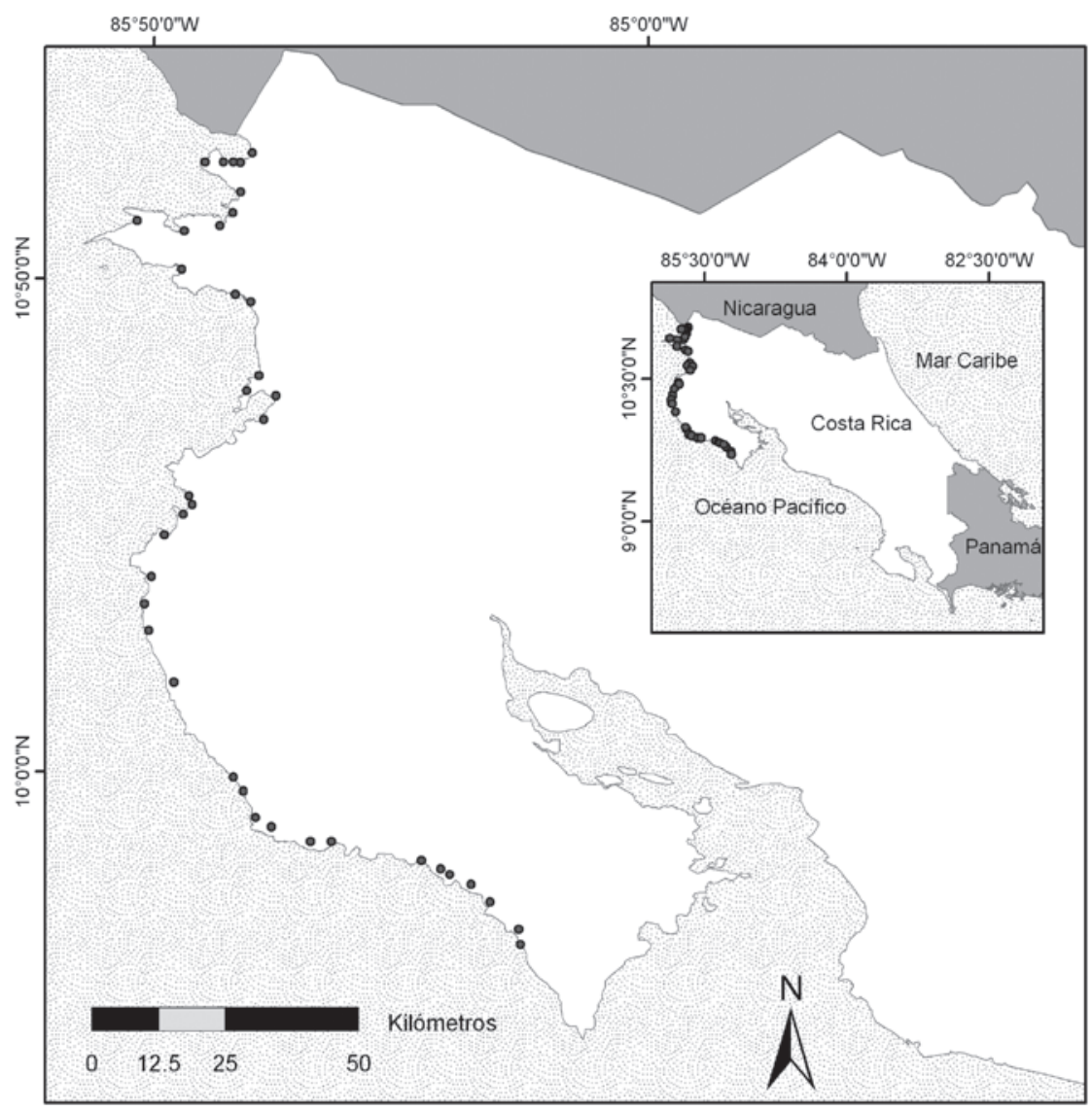

Fig. 1. Región norte del país con indicación de los manglares y ubicación en Costa Rica.

Fig. 1. North section of the Pacific coast of Costa Rica and location in the country.

Parámetros abióticos: La precipitación anual promedio de la zona es de $1978 \mathrm{~mm}$, con una temperatura de $25.4^{\circ} \mathrm{C}$, y se ubica en la zona tropical seca (Tosi 1969, Soto \& Jiménez 1982) (788 mm y $26.8{ }^{\circ} \mathrm{C}$ según Tejada-Rivas 2002). Soto \& Jiménez (1982) observaron un gradiente de salinidad intersticial muy marcado y común de zonas áridas, determinado por la periodicidad del aporte de agua dulce, el bajo caudal de los tres riachuelos que irrigan la cuenca y una estación seca bien definida y prolongada. Adicionalmente, es posible que la alteración del área en el pasado para salinas influya en los regímenes actuales (R. Soto com. pers. 2007).
Entre 1979 y 1981, Soto \& Jiménez (1982) determinaron un gradiente de salinidad que iba de 35 PSU en el margen del canal a 8 PSU en el límite de la vegetación de agua dulce y hasta 163 PSU en el centro del salitral. La distribución de especies de mangle, según los autores, se encuentra en estrecha relación con este gradiente de salinidad.

Descripción de la vegetación: Los estudios realizados en el manglar de Puerto Soley se concentran exclusivamente en la descripción de su vegetación. Soto \& Jiménez (1982) mencionan la presencia de Rhizophora mangle y Rhizophora harrisoni a la orilla de los canales 


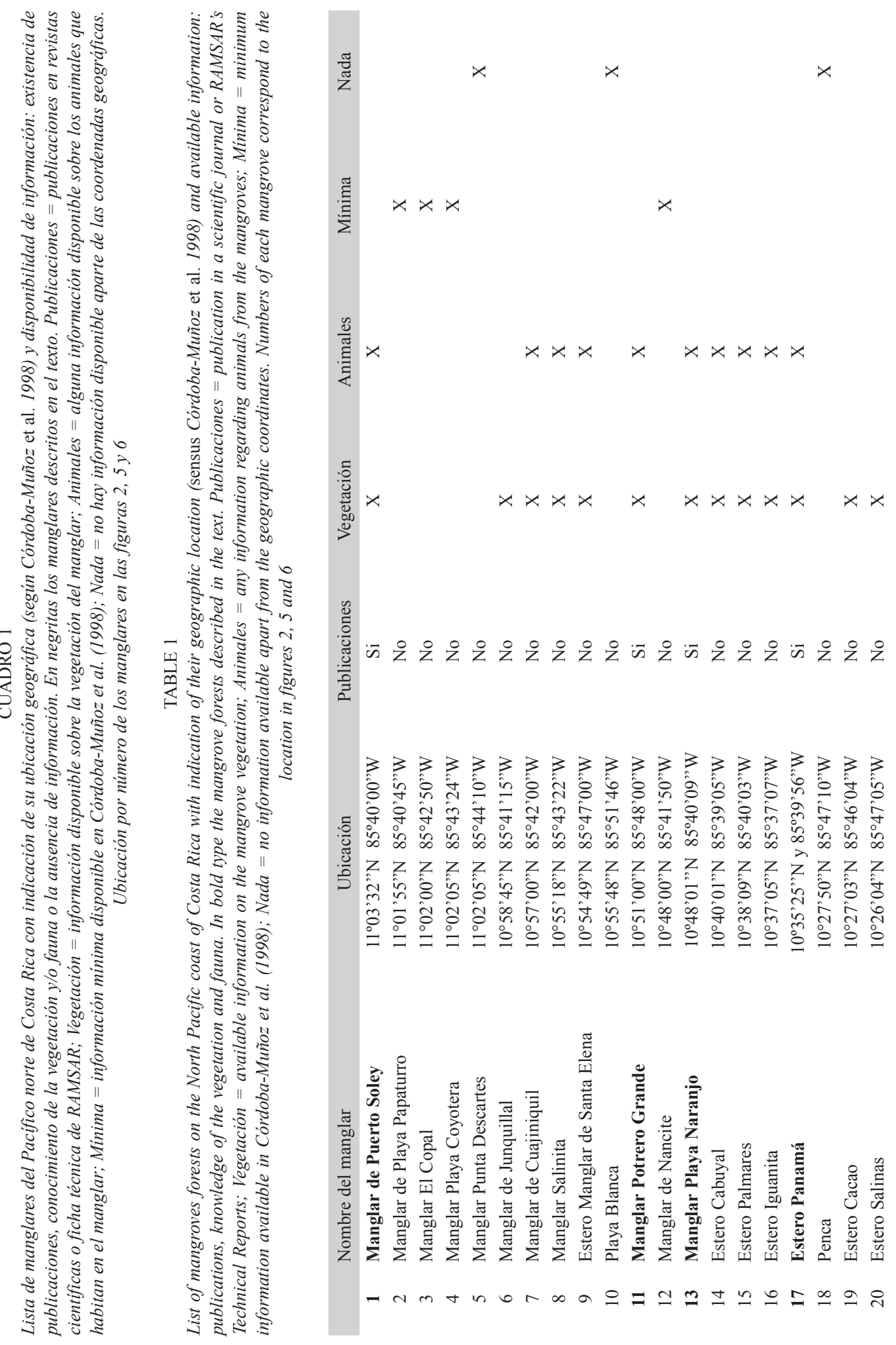




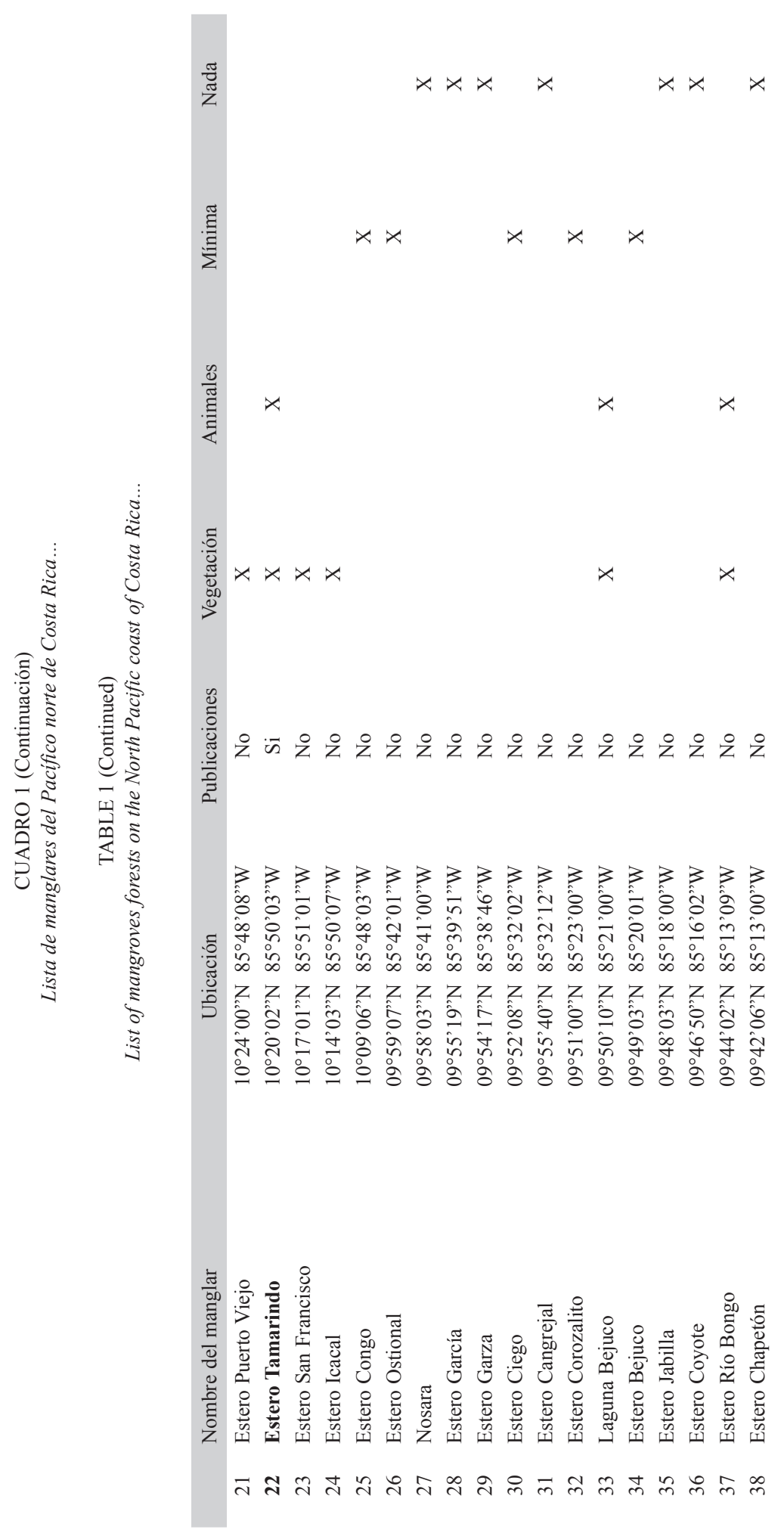




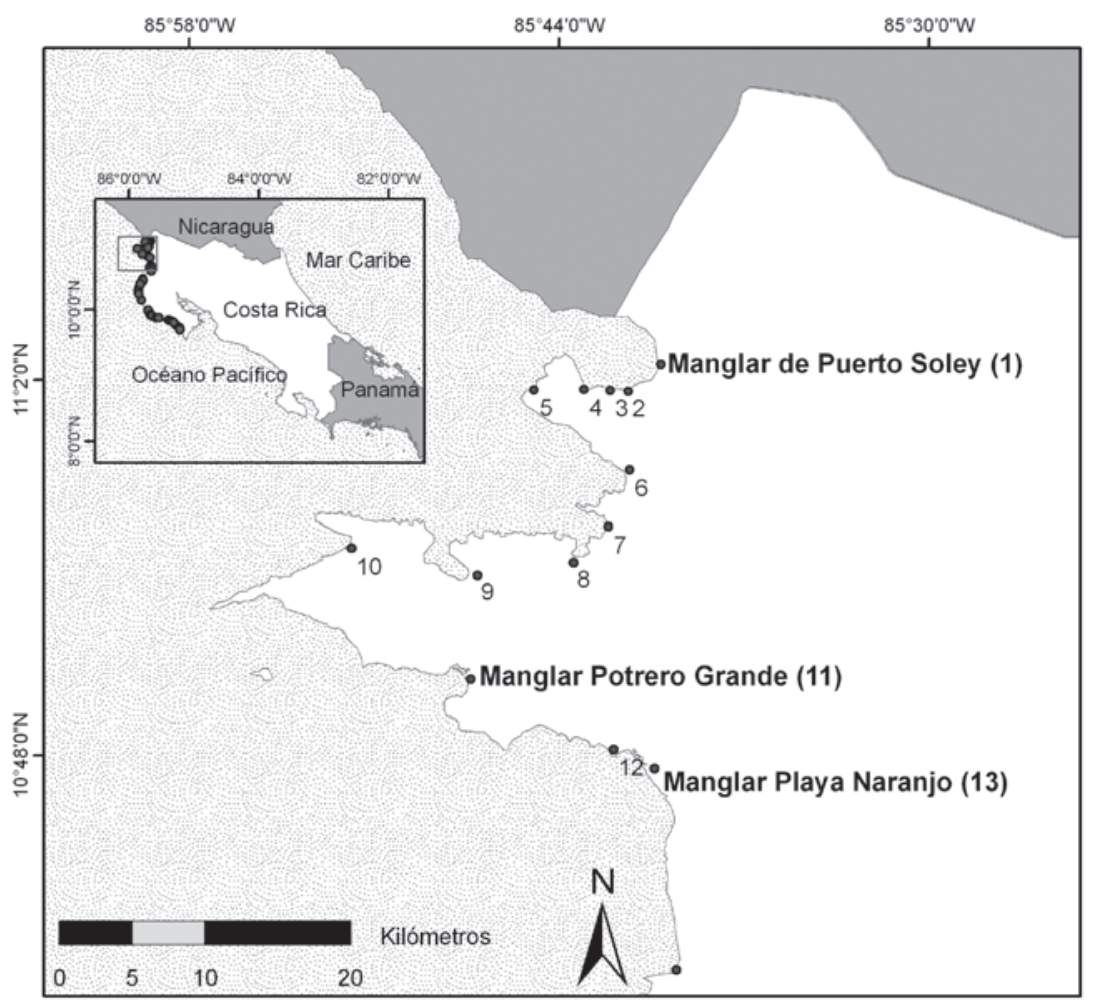

Fig. 2. Extremo norte de la costa Pacífica de Costa Rica. Los manglares con nombres corresponden a los descritos en el texto. Cada número corresponde a un manglar indicado en el Cuadro 1.

Fig. 2. Northern most section of the Pacific coast of Costa Rica. The mangrove forest indicated by name are described in the text. Each number corresponds to a mangrove listed in Table 1.

y en una franja de $40 \mathrm{~m}$ de ancho. En otra zona localizada tierra adentro la especie dominante es Avicennia germinans. Esta última zona está bordeada por individuos de $A$. germinans de alturas menores a los $25 \mathrm{~cm}$ (Fig. 3) y rodeada por un salitral sin vegetación. Esta conformación cambia tierra adentro por individuos más grandes de otras especies como Avicennia bicolor, Conocarpus erectus y Laguncularia racemosa. Los cuales a su vez se mezclan en las áreas cubiertas durante la pleamar y a la orilla de los canales con Capparis odoratissima y Tamarindus indica, especies que forman parte de la vegetación marginal facultativa. Lejos de la pleamar el manglar se torna menos denso y otras especies asociadas dominan la vegetación
(Cuadro 2). Esta distribución es producto del gradiente de salinidad observado en el manglar donde $A$. germinans domina en los sitios de más alta salinidad (promedio de 100.5 PSU), mientras Rhizophora se encuentra más hacia las orillas del canal donde la salinidad promedio tiene un valor menor (57.5 PSU). La alta salinidad resulta en la reducción en la altura, el área basal y el tamaño de las hojas (Soto \& Jiménez 1982, Jiménez \& Soto 1985, Soto \& Corrales 1987).

Avicennia germinans es una especie que está muy bien adaptada para tolerar altos niveles de salinidad (Jiménez \& Soto 1985). Soto \& Corrales (1987) reportan una importante variación fenotípica en la forma y tamaño de 


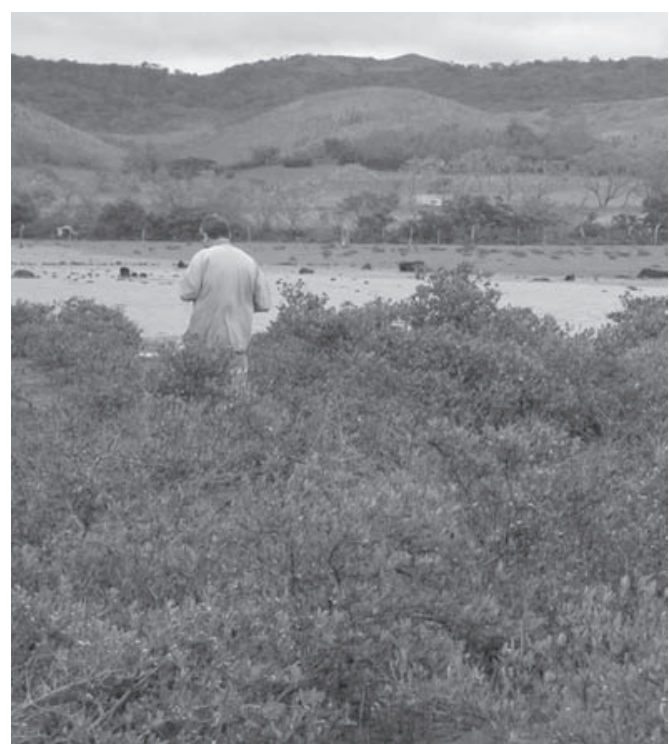

Fig. 3. Mangle enano, Avicennia en el manglar de Puerto Soley. Fotografía por Ulrich Saint-Paul.

Fig. 3. Dwarf mangrove, Avicennia in the mangrove at Puerto Soley. Photograph by Ulrich Saint-Paul.

las hojas de esta especie, asociada con un gradiente climático y salino. Las hojas presentan una menor variabilidad en longitud, ancho y área foliar conforme aumenta la salinidad y disminuye la altura de los árboles.

Las características áridas de este manglar facultan también la presencia de especies vegetales comunes en playas arenosas, como lo es Uniola pittieri, especie presente solo en manglares donde los suelos son arenosos e inundados durante las pleamares (Jiménez \& Soto 1985). Adicionalmente, el bosque presenta un índice de complejidad general bastante más bajo que otros manglares con mayor precipitación y escorrentía (Jiménez \& Soto 1985).

En un estudio realizado sobre la distribución de algas en los manglares del Pacífico costarricense se reportan cuatro especies para el manglar de Puerto Soley: las algas rojas Bostrychia pinnata (Rhodomelaceae), Caloglossa stipitata (Delesseriaceae) y Caloglossa leprieurii (Delesseriaceae), y el alga verde Rhizoclonium
CUADRO 2

Ubicación de la vegetación marginal y marginal facultativa del manglar de Puerto Soley, Guanacaste. Fuente: Soto y Jiménez (1982)

TABLE 2

Location of marginal and facultative marginal vegetation in Puerto Soley mangrove, Guanacaste. Source Soto and Jiménez (1982)

\begin{tabular}{ll}
\multicolumn{1}{c}{ Especie } & Ubicación \\
Randia sp. & lejos de la pleamar \\
Sesuvium portulacastrum & bajo efecto de la pleamar \\
Blutaparon vermiculare & bajo efecto de la pleamar \\
Jouvea straminea & bajo efecto de la pleamar \\
Fimbristilys spadicea & suelos no salinos \\
Heliotropium curassavicum & suelos no salinos \\
Loeselia cliliata & suelos no salinos \\
Caparis odoratissima & suelos no salinos \\
Tevethia ovata & suelos no salinos \\
Ximenia americana & suelos no salinos \\
Egletes viscosa & suelos no salinos \\
Turnera ulmifolia & suelos no salinos \\
Jouvea straminea & suelos no salinos/límite \\
Canavalia maritima & marea alta \\
Ipomoea pescaprae & límite marea alta \\
Caesalpinia & límite marea alta \\
Prosopis juliflora & límite marea alta \\
& límite marea alta
\end{tabular}

riparium (Cladophoraceae). De ellas la especie más abundante y cosmopolita fue $B$. pinna$t a$, que se encontró en todos los sectores del manglar y en todas las secciones de las raíces analizadas (Tejada-Rivas 2002).

\section{Manglar de Potrero Grande}

Ubicación: Se ubica en las coordenadas $10^{\circ} 50^{\prime} 44^{\prime \prime} \mathrm{N}$ y $86^{\circ} 46^{\prime} 45^{\prime \prime} \mathrm{W}\left(10^{\circ} 51^{\prime} 00^{\prime} \mathrm{N}\right.$ 8548'00"W en Córdoba-Muñoz et al. 1998), en la Península de Santa Elena, Guanacaste y posee una cobertura de alrededor de 139 ha (Fig. 2). Forma parte de la zona de vida Bosque Seco Tropical y su principal vía de acceso es la marina (Córdoba-Muñoz et al. 1998). 
Parámetros abióticos: La precipitación anual oscila entre los 1400-1500 mm, y el clima se caracteriza por una estación muy seca y caliente (Córdoba-Muñoz et al. 1998). El manglar es irrigado por los ríos Potrero Grande y Boquerones (Córdoba-Muñoz et al. 1998). No hay datos sobre las características físico-químicas del agua o de los sedimentos del manglar.

Descripción de la vegetación: Este es considerado como el manglar de bosque seco más desarrollado (Fig. 4) y en mejor estado de la costa Pacífica de Costa Rica. Único además, por la existencia de una población saludable de la especie de Pelliciera rhizophorae, muy poco abundante en la región (Jiménez \& Soto 1985, Ramsar 1999). Estas características especiales le han conferido desde 1999 el status de sitio de importancia internacional según la Convención de Humedales Ramsar (Ramsar 1999).

Las vegetación nuclear está compuesta por las especies $R$. racemosa, A. germinans, $A$. bicolor, L. racemosa y P. rhizophorae (Ramsar 1999). Especies como Fimbristylis spadice, Sesuvium portulacastrum, Ipomoea pes-caprae y $C$. erecta son algunas de las especies marginales del manglar (Córdoba-Muñoz et al. 1998).

Descripción de la fauna: En este manglar se encuentran bivalvos como Anadara tuberculosa, Anadara multicostata, Crassostrea corteziensis y Grandiarca grandis (Ramsar 1999), gasterópodos como Littorina zebra y Cerithidea valida, y otros grupos animales como los crustáceos de los géneros Uca y Penaeus (Córdoba-Muñoz et al. 1998).

\section{Manglar de Playa Naranjo}

Ubicación: Se ubica dentro del área protegida Parque Nacional Santa Rosa, específicamente en la parte baja detrás de Playa Naranjo y ocupa aproximadamente 80 ha; se conoce también como Manglar de Santa Rosa. Se localiza entre los $10^{\circ} 48^{\prime} 01^{\prime \prime} \mathrm{N}$ y $85^{\circ} 40^{\prime} 09^{\prime}$ 'W

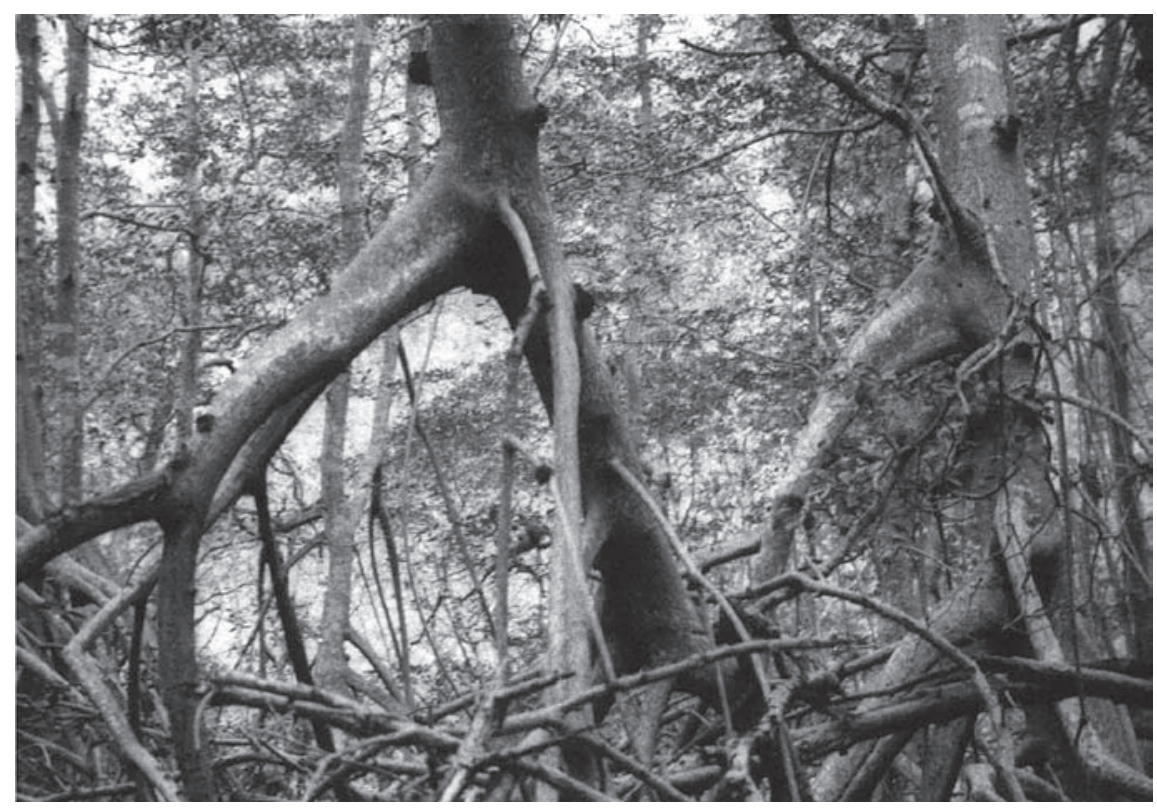

Fig. 4. Manglar de Potrero Grande, Área de Conservación Guanacaste. Fotografía por Percy Denyer.

Fig. 4. Mangrove forest at Potrero Grande, Área de Conservación Guanacaste. Photograph by Percy Denyer. 
(Bravo \& Rivera 1998, Córdoba-Muñoz et al. 1998) (Fig. 2). La sección tierra adentro colinda con un bosque anegado de vegetación mixta (Bravo \& Rivera 1998).

Putz et al. (1984) mencionan otro manglar dentro del Parque Nacional Santa Rosa, el cual por la descripción dada aunque no la ubicación $\left(10^{\circ} 35^{\prime} \mathrm{N}\right.$ y $85^{\circ} 85^{\prime} \mathrm{W}$; este último número está consignado erróneamente en la publicación) pareciera que corresponde al manglar de Santa Rosa. Algunos aspectos descritos coinciden con los datos de Bonoff \& Janzen (1980) para el manglar de Santa Rosa. Por lo que podría tratarse de al menos una región contigua, razón por la cual se incluyen los datos de Putz et al. (1984) en esta sección.

Parámetros abióticos: Este manglar no está delimitado en la hoja cartográfica 1:50 000 (Ahogados), pero según su ubicación se podría sugerir que el río Nisperal y el río Calera son las quebradas intermitentes que lo irrigan con agua dulce (IGNCR 1988a). Durante un mismo mes, este manglar puede ser inundado o no por la marea alta en un ciclo de inundación de 24 horas (Bonoff \& Janzen 1980), dependiendo de la amplitud de la marea que tiene un promedio de $2.29 \mathrm{~m}$ (Pool et al. 1977). El suelo fangoso está cubierto casi por completo de neumatóforos de Avicennia y se puede mantener muy húmedo o seco dependiendo de la sombra que proporcionan los árboles (Bonoff \& Janzen 1980). En donde el suelo es arenoso la especie dominante es $R$. mangle (Pool et al. 1977).

Putz et al. (1984) reportaron para la época seca una velocidad del viento de $11 \mathrm{~m} / \mathrm{s}$ con periodos de calma. En 1973 Pool et al. (1977) registraron un promedio de precipitación anual de $1800 \mathrm{~mm}$ y $25.0{ }^{\circ} \mathrm{C}$ de temperatura atmosférica. La época lluviosa va desde junio a noviembre, mientras que la época seca se extiende desde diciembre a mayo. Por lo que pertenece a la zona de vida tropical seca (Holdrigde et al. 1971).

Descripción de la vegetación: La vegetación dominante incluye $R$. mangle, $R$. racemosa,
A. germinans y A. bicolor. En el sector fangoso la mayoría de la vegetación nuclear está representada por rodales monoespecíficos de $A$. germinans (informado como Avicennia nitida por Bonoff \& Janzen 1980, y por Putz et al. 1984). En estos rodales se describe la existencia de espacios entre copas con filtración de luz debido a la abrasión mecánica entre las ramas y hojas que causa una poda recíproca entre los árboles cercanos (Putz et al. 1984). Cerca del estuario se observan también algunos pocos individuos de $A$. germinans (informado como A. tonduzii por Putz et al. 1984), C. erectus y $R$. mangle cerca del estuario (Bonoff \& Janzen 1980, Putz et al. 1984).

Pool et al. (1977) describen un área del manglar en donde Rhizophora sp. se ubica en un bosque franjeante que recibe poca acción del oleaje. Seguido por Avicennia sp. y posteriormente cactus y otras especies xerofíticas. Para este manglar se indica un área basal de $2.32 \mathrm{~m}^{2} / 0.1$ ha para árboles mayores de $2.5 \mathrm{~cm}$ de DAP. El índice de complejidad calculado fue de 4.9 que es típico para zonas muy áridas (Jiménez \& Soto 1985). Se menciona además que en este sector el género con el mayor valor de importancia fue Rhizophora sp. seguida por Laguncularia. En este manglar, Rodgers y Horn (1996) realizaron el único estudio en el país sobre polen de manglar. Las muestras, recolectadas en un rodal monoespecífico de Avicennia, mostraron que los espectros de polen de Rhizophora y Avicennia son buenos indicadores de la presencia de mangle en una zona. Sin embargo, debido a que Avicennia deposita menor cantidad de polen que Rhizophora la abundancia de estas especies podría subestimarse.

Descripción de la fauna: Este es el único manglar de Costa Rica en el que se ha hecho un estudio de roedores que lo habitan. Desafortunadamente, este es el único grupo animal descrito para este sistema. Al comparar las diferentes especies de roedores presentes en los distintos hábitats del Parque Nacional Santa Rosa, Oryzomys palustris fue el roedor que se capturó con mayor frecuencia en la zona 
de manglar, se considera que es debido a su habilidad para escalar y porque además se puede alimentar de cangrejos (Bonoff \& Janzen 1980). Sigmodon hispidus también fue capturado en el sitio, pero en menor cantidad. Bonoff \& Janzen (1980) especulan que debido a las condiciones particulares de un manglar es probable que estos roedores utilicen nidos arbóreos.

\section{Área de Conservación Tempisque}

\section{Manglar de Estero Panamá}

Ubicación: Se ubica en la parte trasera de Playa Panamá en Bahía Culebra, Guanacaste, $10^{\circ} 35^{\prime} 25^{\prime \prime} \mathrm{N}$ y $85^{\circ} 39^{\prime} 56^{\prime \prime} \mathrm{W} \quad\left(10^{\circ} 30^{\prime} 09^{\prime \prime} \mathrm{N}\right.$ $85^{\circ} 45^{\prime} 00^{\prime \prime} \mathrm{W}$ según Cabrera-Peña et al. 1994). Tiene una cobertura estimada para 1998 de 60 ha (Bravo \& Rivera 1998) (Fig. 5).

Parámetros abióticos: Este manglar es irrigado por ríos intermitentes o quebradas (IGNCR 1988b), no hay publicaciones que describan los aspectos físico-químicos de este sistema.

Descripción de la vegetación: Es tan escasa la información sobre este manglar que ni siquiera aparece delimitado en el mapa 1:50 000 (IGNCR 1988b). Sin embargo, Cabrera-Peña et al. (1994) mencionan que están presentes los

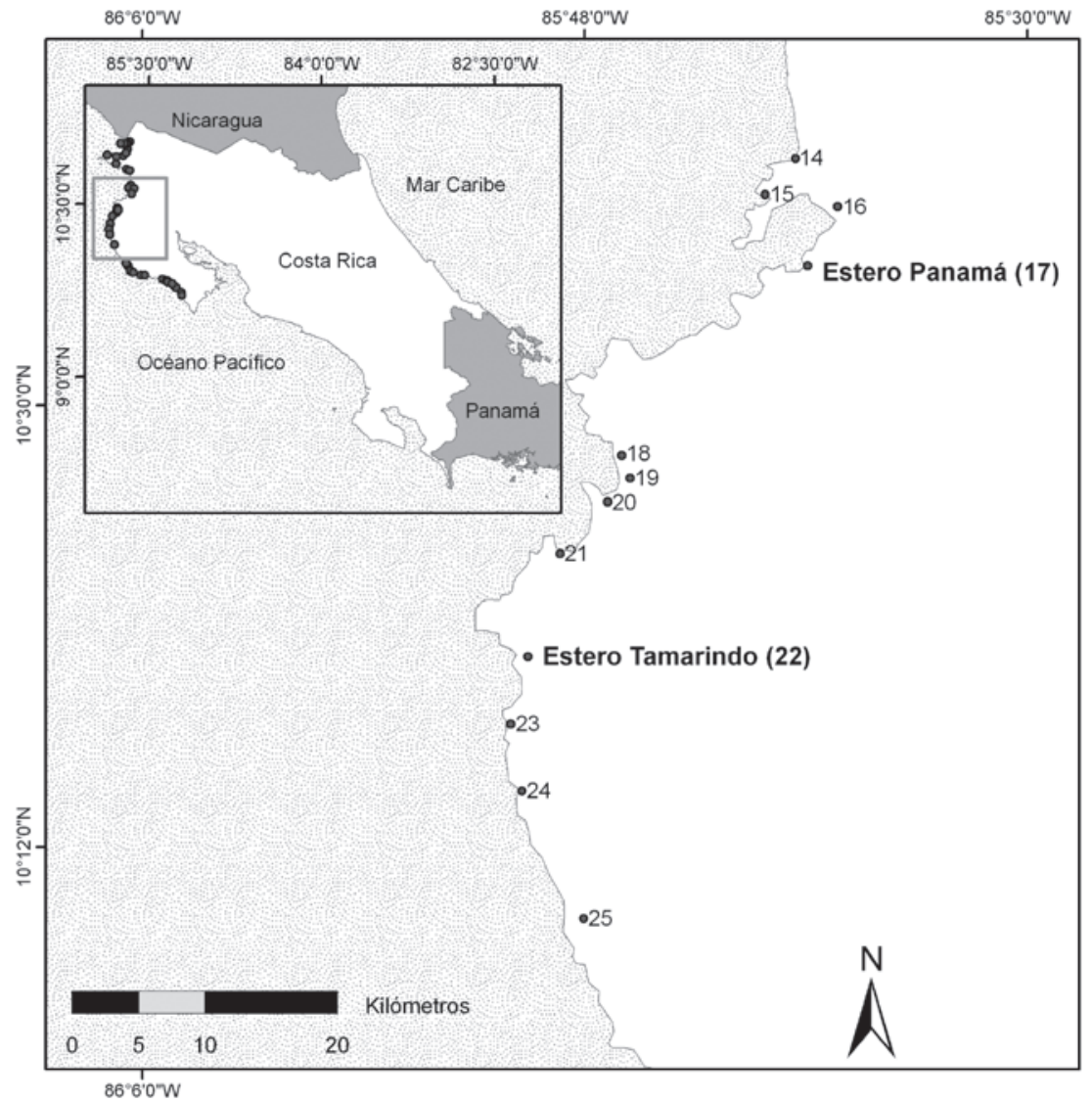

Fig. 5. Sección de la costa Pacífica entre Bahía Culebra y Junquillal. Los manglares con nombres corresponden a los descritos en el texto. Cada número corresponde a un manglar indicado en el Cuadro 1.

Fig. 5. Section of the Pacific coast between Bahía Culebra and Junquillal. The mangrove forest indicated by name are described in the text. Each number corresponds to a mangrove listed in Table 1. 
géneros Rhizophora, Avicennia y Laguncularia, pero no indican cuáles especies.

Descripción de la fauna: Cabrera-Peña et al. (1994) indican los tamaños y la proporción sexual del cangrejo Ucides occidentalis. Este cangrejo posee madrigueras simples con caverna terminal sin interconexiones. La población tiene una mayoría de machos entre las tallas de 51.0-56.0 $\mathrm{mm}$ y una mayoría de hembras entre los 46.0-51.0 mm, y están en una proporción de aproximadamente dos machos por cada hembra (Cabrera-Peña et al. 1994).

\section{Manglar de Tamarindo}

Ubicación: Localizado en el cantón de Santa Cruz, Guanacaste, en los 10¹7'30' N y 8549'00"W (Ramsar 1993, Ley 7149 1990) (10²0'02”N 85'50'03'W en Córdoba-Muñoz et al. 1998) (Fig. 5) y es la formación de manglar más grande en la parte externa del Golfo de Nicoya (Pizarro \& Angulo 1993, Bravo \& Rivera 1998). Es parte del Refugio Nacional de Vida Silvestre Tamarindo (desde 1990) y desde 1993 es considerado sitio de importancia internacional por la Convención sobre Humedales Ramsar (Ramsar 1993). El manglar posee una extensión aproximada de 400 ha, con un área costera constituida principalmente por arenas finas y al interior de la línea costera se encuentra plantas herbáceas (Ramsar 1993).

Parámetros abióticos: Tamarindo se ubica dentro de una región seca del Pacífico costarricense, con un promedio de precipitación anual de $2031 \mathrm{~mm}$ y una temperatura promedio de $25.4^{\circ} \mathrm{C}$ (Jiménez \& Soto 1985 , Pizarro \& Angulo 1993). Los ríos Matapalo y San Andrés son dos de los principales ríos que desembocan en este manglar (Bravo \& Rivera 1998), con un área de cuenca de 84.3 $\mathrm{km}^{2}$ (Jiménez \& Soto 1985). Sin embargo, se desconocen los valores de salinidad, oxígeno y $\mathrm{pH}$ del agua que inunda el manglar. Jiménez (1981) hizo análisis de suelo y reportó un ámbito de salinidad de 32-51 PSU y de contenido de materia orgánica de 13.4-24.4\%. El canal principal del estero es de más de $2 \mathrm{~km}$ de largo y se llega a dividir en dos o tres canales secundarios (MINAE s/f).

Descripción de la vegetación: La vegetación nuclear está compuesta por cinco especies, $R$. racemosa, A. bicolor, A. germinans, $R$. mangle y $P$. rhizophorae. Esta última especie rara vez encontrada en manglares con altas salinidades como las del Pacífico norte, lo que explica su baja abundancia (Jiménez 1984, Jiménez \& Soto 1985). Al igual que en otras formaciones del Pacífico, $R$. mangle se ubica en la franja más cercana del canal y se mezcla con $R$. racemosa tierra adentro. En el frente del manglar se encuentran las menores salinidades y $R$. mangle se entremezcla también con P. rhizophorae (Pizarro \& Angulo 1993). En el interior del manglar, donde los valores de salinidad son máximos, se ubican los rodales de $A$. germinans y $A$. bicolor. Siendo estas dos especies junto con $R$. racemosa las que presentan los valores de dominancia relativa más altos (Jiménez 1981). Se han registrado árboles con tamaños de hasta $25-30 \mathrm{~m}$ de altura y $45 \mathrm{~cm}$ de diámetro (MINAE s/f).

Descripción de la fauna: La ficha técnica Ramsar (1993) reporta la presencia de las aves: Phalacrocorax olivaceus, Fregata magnificens, Buteogallus anthracinus, Buteo magnirostris y Pandion hallaetus, y los mamíferos: Vampirum spectrum, Allouatta palliata y Didelphis marsupiales. La información turística se refiere al sitio como con presencia de una avifauna relativamente diversa, así como de monos y lagartos (Franco-Mata 1999).

\section{Otros manglares de la región}

Aparte de los cinco manglares descritos anteriormente existen otros manglares en la región de los cuales no se encontró información que nos permitiera describirlos con cierto detalle. De los manglares de Junquillal, Cuajiniquil, Salinita, Estero Manglar de Santa Elena, Estero Cabuyal, Estero Palmares, Estero Iguanita, Estero Cacao, Estero Salinas, Estero Puerto 
Viejo, Estero San Francisco, Estero Icacal, Laguna Bejuco y Estero Río Bongo (Figs. 1, 2, 5, 6 y Cuadro 1) solo se conoce la lista de las especies vegetales nucleares y marginales más importantes, así como la lista de la fauna más importante de algunos de estos manglares (Córdoba-Muñoz et al. 1998). De los nueve manglares restantes solo se conoce su ubicación (Figs. 1, 2, 5, 6 y Cuadro 1).

\section{DISCUSIÓN Y CONCLUSIONES}

De los 38 manglares localizados en el Pacífico norte de Costa Rica (Fig. 1) solamente cinco han sido descritos en 17 publicaciones (13 artículos en revistas científicas, dos capítulos de libros y dos tesis de maestría) y dos fichas técnicas de Ramsar. La mayoría de las publicaciones con información primaria se realizaron en la primera mitad de la década de 1980, sin embargo la mayoría de los documentos, inventarios de toda la costa y fichas técnicas son de la década de 1990. (Cuadro 3). La primera publicación científica fue por Pool et al. (1977) en la que describen el manglar de Santa Rosa. Antes de esa fecha, William Beebe, hace una descripción anecdótica de los árboles de mangle debajo de los que se sentó a escribir en Bahía de Santa Elena (Beebe 1942). Las dos últimas publicaciones realizada para los manglares de la región son la tesis de Tejada-Rivas (2002), donde describe la relación entre las

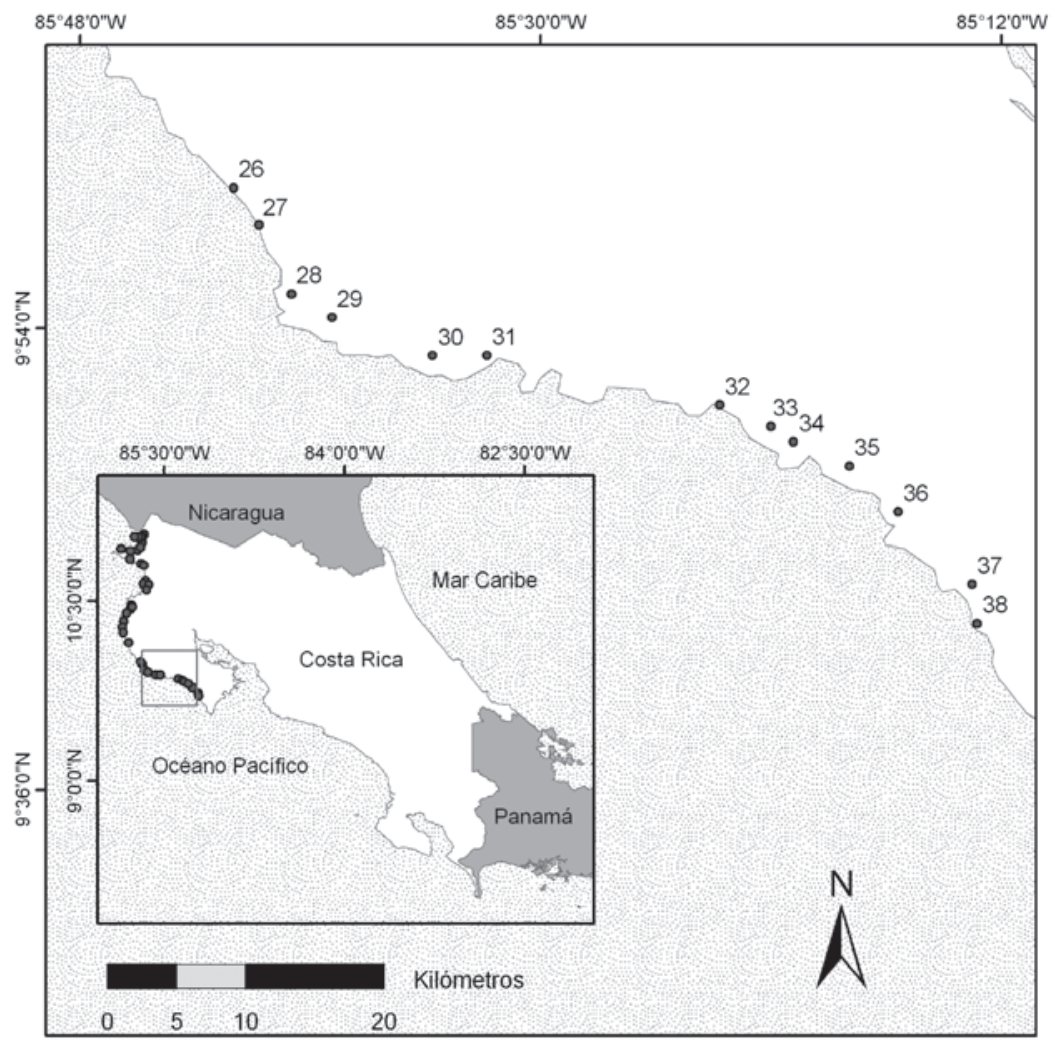

Fig. 6. Sección de la costa Pacífica entre Junquillal y Manzanillo de Ario. Cada número corresponde a un manglar indicado en el Cuadro 1.

Fig. 6. Section of the Pacific coast between Junquillal and Manzanillo de Ario. The mangrove forest indicated by name are described in the text. Each number corresponds to a mangrove listed in Table 1. 
CUADRO 3

Número de publicaciones, tesis e informes realizados por quinquenio sobre los manglares

del Pacifico norte de Costa Rica

TABLE 3

Number of publications, theses and reports made on mangroves every five years in the Norht Pacific, Costa Rica

$\begin{array}{cccccccc} & \text { Antes de } & 1976- & 1981- & 1986- & 1991- & 1996- & 2001- \\ & 1975 & 1980 & 1985 & 1990 & 1995 & 2000 & 2005 \\ \text { Número de publicaciones } & 0 & 2 & 3 & 1 & 5 & 5 & 2\end{array}$

algas y el bosque de manglar de Puerto Soley, y un capítulo sobre los manglares de la región seca de Costa Rica por Jiménez (2004).

La mayoría de las publicaciones, siete de ellas, son sobre inventarios de los manglares de Costa Rica o Centro América. La distribución de la flora del manglar es el tema más desarrollado con cuatro artículos (Pool et al. 1977, Putz et al. 1977, Soto \& Jiménez 1982, Jiménez \& Soto 1985), y además, uno sobre el polen de los mangles (Rodgers \& Horn 1996) y una tesis sobre las algas asociadas a raíces de mangle (Tejada-Rivas 2002). Sobre animales hay tres trabajos, uno sobre roedores (Bonoff \& Janzen 1980), otro sobre una especie de cangrejo (Cabrera-Peña et al. 1994) y uno sobre moluscos de importancia comercial de los manglares del Pacífico de Costa Rica (Cruz 1999). El otro tema tocado es un capítulo sobre turismo en manglares de Costa Rica (FrancoMata 1999). Los trabajos sobre toda la costa predominan con 10 capítulos o libros. Los dos manglares más estudiados del Pacífico norte son el de Puerto Soley y el de Santa Rosa, cada uno con tres artículos en revistas científicas; del manglar de Playa Panamá hay un artículo. De los otros manglares hay fichas técnicas de Ramsar o informes, tres de Tamarindo y una de Potrero Grande. Claramente hace falta estudiar más manglares y en más detalle en la región del Pacífico norte de Costa Rica, especialmente por la presión a que están siendo sometidos por el desarrollo turístico.

En el caso de los datos físico-químicos existentes estos son de hace muchos años.
Los análisis de sustratos, de suma importancia para explicar la distribución del bosque son escasos. La dinámica trófica de los sistemas no ha sido estudiada y mucho menos los aspectos relacionados con la productividad del mismo, la exportación de nutrientes y la producción de los mismos a través del fitoplancton. Faltan datos actualizados sobre la cobertura y desarrollo estructural y mapas detallados del bosque de manglar. Estos son algunos de los campos que han sido prácticamente inexplorados y necesitan ser analizados de manera tal que permitan entender mejor a los manglares de la región y determinar los cambios que han ocurrido a través del tiempo en los sitios ya estudiados.

Macintosh \& Ashton (2004) proponen que la investigación científica en los manglares es uno de los principios para el desarrollo de un Código de Conducta para la Gestión y uso Sostenible de Ecosistemas de Manglar. Los autores aseguran que "todas las decisiones referentes a las acciones de desarrollo deberán ser formuladas con base a un amplio cimiento de conocimientos que deberían incluir evaluaciones a escala de paisaje o regiones sobre la capacidad de los recursos". Por lo que aseguran que uno de los principales obstáculos para la conservación y gestión sostenible de los ecosistemas de manglar es la comprensión inadecuada de las funciones y de los valores de estos ecosistemas.

Si se desea conservar los manglares se debe generar información que permita conocer el tamaño crítico que se requiere para que un manglar mantenga sus funciones básicas, 
comprender las diversas interacciones que ocurren entre las pesquerías y el manglar, los patrones de flujo de nutrientes, la productividad de los manglares, los diversos impactos que las actividades antropogénicas han causado en los mismos, su biodiversidad tanto de organismos terrestres como de organismos acuáticos, así como la realización de estudios multidisciplinarios que permitan realizar una valoración de los beneficios económicos de los ecosistemas de manglar, como también documentar los sistemas de gestión tradicionales en los manglares (Macintosh \& Ashton 2004). Otros estudios que deberían emprenderse en los manglares no solo del norte pero de todo el país, son aspectos de circulación de las aguas, temas biogeoquímicos y más trabajo sinóptico en cuanto a la variación de la cobertura de vegetación de mangle a través del tiempo (R. Lara com. pers. 2003).

El conocimiento de los manglares del Pacífico Norte de Costa Rica es apenas incipiente, pero justo para mostrar un panorama a grandes rasgos de los manglares que tenemos en la región y su distribución actual. Sin embargo, falta mucho por conocer, en muchos de los manglares no se ha iniciado la investigación básica, e.i. inventarios de especies y en aquellos manglares en los que se ha generado cierto conocimiento es necesario profundizar en los temas de investigación y generar conocimiento que permita conocer la dinámica de estos complejos sistemas biológicos. Trabajos de mapeo detallado, caracterización de la vegetación y la fauna, estudios fisiológicos, análisis de procesos biogeoquímicos, evaluaciones económicas, y determinación del estado de salud de los manglares del Pacífico norte de Costa Rica, y del resto del país son necesarios y urgentes.

\section{AGRADECIMIENTOS}

Priscilla Zamora Trejos desapareció mientras buceaba en la Isla Malpelo, Colombia, el 10.II.2006. Priscilla había avanzado bastante con esta publicación e iniciado otras sobre los manglares de otras partes del país. Me ha tocado a mí tratar de terminarlas. Esta publicación se la dedico a la familia de Priscilla.
Se le agradece a la Vicerrectoría de Investigación, Universidad de Costa Rica (proyecto 808-A3-518), al CONICIT (FV-046-02) y a la PADI Foundation por el apoyo económico. Al señor $\mathrm{M}$. Tupper por su apoyo a las investigaciones marinas en Costa Rica. También se le agradece a todas las personas que colaboraron en la búsqueda de bibliografía. A Catalina Benavides y Damaris Torres-Pulliza por su ayuda con los mapas. Finalmente le agradecemos a Ricardo Soto, Jorge Jiménez, Margarita Silva, José Antonio Vargas, Jimena Samper y Damaris Torres-Pulliza la revisión de una versión anterior de este manuscrito.

\section{RESUMEN}

Costa Rica tiene bosques de manglar en las costas Caribe y Pacífica. El 99\% de los manglares del país se encuentran en la costa Pacífica. En este trabajo recopilamos la información disponible sobre los manglares del Pacífico norte de Costa Rica, desde la frontera con Nicaragua hasta la Reserva Absoluta de Cabo Blanco, en la punta sur de la Península de Nicoya. La ubicación de los manglares y cualquier información disponible para cada manglar es resumida. El clima de esta región es seco con estaciones de lluvia y sequía muy bien definidas. Los manglares del norte son relativamente pequeños en extensión y tamaño de árboles, y de baja diversidad comparados con los del Pacífico sur de Costa Rica. Las principales especies de mangle son: Rhizophora mangle, Rhizophora racemosa a lo largo de los bordes de los canales, seguido por Avicennia germinans, y más tierra adentro Avicennia bicolor, Laguncularia racemosa y Conocarpus erectus. En el manglar de Potrero Grande se informa de una población saludable de la especie de Pelliciera rhizophorae, especies muy poco abundante. Reconocemos 38 comunidades de manglares en el Pacífico norte de Costa Rica basado en el Inventario Nacional de Humedales, artículos publicados, observaciones de campo, tesis, informes técnicos y los mapas 1: 50000 del Instituto Geográfico Nacional. Se pudo encontrar información relativamente detallada pero fragmentada de únicamente cinco manglares (los descritos en esta publicación), de 14 solamente hay listas preliminares e incompletas de plantas y en algunos casos de animales, de nueve manglares más hay información todavía más limitada, y de otros nueve se conoce solamente su ubicación, que en algunos casos se comprobó que era incorrecta. Trabajos de mapeo detallado, caracterización de la vegetación y la fauna, estudios fisiológicos, análisis de procesos biogeoquímicos, evaluaciones económicas, y determinación del estado de salud de los manglares del Pacífico norte de Costa Rica, y del resto del país son necesarios y urgentes. 
Key words: Manglares, Costa Rica, Pacífico tropical este, impacto humano, Guanacaste.

\section{REFERENCIAS}

Beebe, W. 1942. Book of Bays. Harcourt, Brace, Nueva York.

Bilio, M., A. Saborío Coze \& A. Hernández Potocarrero. 1999. Los manglares, los camarones y el desarrollo de las áreas costeras en el Istmo Centroamericano. EC Fish. Coop. Bull. 12: 18-20.

Bravo, J. \& L. Rivera. 1998. Mapas de Humedales de Costa Rica e Información Complementaria. SINAC, MINAE -UICN. Escala 1:200 000.

Bonoff, M.B. \& D.H. Janzen. 1980. Small terrestrial rodents in eleven habitats in Santa Rosa National Park, Costa Rica. Brenesia 17: 163-174.

Cabrera-Peña, J., F. Vives-Jiménez \& Y. Solano-López. 1994. Tamaños y proporción sexual de Ucides occidentales (Crustacea: Gecarcinidae) en un manglar de Costa Rica. Uniciencia 11: 97-99.

Castaing, A., J.M Jiménez \& C.R. Villalobos. 1980. Observaciones sobre la ecología de manglares de la Costa Pacífica de Costa Rica y su relación con la distribución del molusco Geloina inflata (Philippi) (Pelecypoda: Corbiculidae). Rev. Biol. Trop. 28: 323-339.

Coll, M., A.C. Fonseca \& J. Cortés. 2001. El manglar y otras asociaciones vegetales de la laguna de Gandoca, Limón, Costa Rica. Rev. Biol. Trop. 49: 321-329.

Córdoba-Muñoz, R., J.C. Romero-Araya \& N.J. Windevoxhel-Lora. 1998. Inventario de los humedales de Costa Rica. UICN, MINAE, SINAC, Embajada Real de los Países Bajos, San José.

Cruz, R.A. 1999. Moluscos de manglar: especies de importancia comercial con posibilidades de manejo: 252-261. In: T. Ammour, A. Imbach, D. Suman \& N. Windevoxhel (Eds.). Manejo productivo de manglares en América Central. Serie Téc. No.7, CATIE, Costa Rica.

Duke, N.C., J.-O. Meynecke, S. Dittman, A.M. Ellison, K. Anger, U. Berger, S. Cannicci, K. Diele, K.C. Ewel, C.D. Field, N. Koedam, S.Y. Lee, C. Marchand, I. Nordhaus \& F. Dahdough-Guebas. 2007. A world without mangroves? Science 317: 41.

Franco-Mata, E. 1999. Turismo en manglares de Costa Rica: una alternativa para mejorar: 159-169. In T. Ammour, A. Imbach, D. Suman \& N. Windevoxhel (Eds.).
Manejo productivo de manglares en América Central. Serie Téc. No. 7. CATIE, Turrialba, Costa Rica.

Germain, N. 2004. Importancia de la calidad de agua del río Conte y recomendaciones para un plan de acción de gestión sostenible Playa Blanca, Golfo Dulce, Costa Rica. Tesis Maestría, GIACT, Universidad de Costa Rica, San Pedro, Costa Rica.

Holdridge, L.R., W.C. Grenke, W.H. Hatheway, T. Liang \& J.A. Tosi, Jr. 1971. Forest Environments in Tropical Life Zones. Pergarmon Press, Nueva York.

Instituto Geográfico Nacional de Costa Rica (IGNCR). 1988a. Mapa 1:50 000, Ahogados, Costa Rica. IGN, San José, Costa Rica.

Instituto Geográfico Nacional de Costa Rica (IGNCR). 1988b. Mapa 1:50 000, Carrillo Norte, Costa Rica. IGN, San José, Costa Rica.

Jiménez, J.A. 1981. The Mangrove of Costa Rica: the physiognomic characterization. Tesis de Maestría, Universidad de Miami, Florida, EEUU.

Jiménez, J.A. 1984. A hypothesis to explain the reduced distribution of the mangrove Pelliciera rhizophorae Tr. \& Pl. Biotropica 16: 304-308.

Jiménez, J.A. 1988. The dynamics of Rhizophora mangle forests on the Pacific coast of Costa Rica. Brenesia 30: $1-12$

Jiménez, J.A. 1994. Bosques de manglares en la Costa Pacífica de América Central. Rev. Forestal Centroamer. 3: 13-17.

Jiménez, J.A. 2004. Mangrove forests under dry seasonal climates in Costa Rica: p. 136-143. In G.W. Frankie, A. Mata \& S.B. Vinson (eds.). Biodiversity Conservation in Costa Rica: Learning the Lesson in a Seasonal Dry Forest. Univ. California Press, Berkely, California.

Jiménez, J.A. \& R. Soto. 1985. Patrones regionales en la estructura y composición florística de los manglares de la costa Pacífica de Costa Rica. Rev. Biol. Trop. 33: 25-37.

Ley 7149. 1990. Refugio Nacional de Vida Silvestre Tamarindo. La Gaceta No 121. San José, Costa Rica.

Macintosh, D. \& A.C. Ashton. 2004. Principios para un código de conducta para la gestión y uso sostenible de ecosistemas de manglar. Banco Mundial, ISME, Aarhus Center, Washington, DC.

MINAE. s/f. Refugio Nacional de Vida Silvestre Tamarindo. Mimeografiado. 
MIRENEM. 1993. Informe de Costa Rica. Taller sobre manejo de humedales y manglares en Centro América y el Caribe, 12-19 de junio 1993, Miami, Florida, EEUU.

Pizarro, F. \& H. Angulo. 1993 Diagnóstico de los manglares de la costa Pacífica de Costa Rica: Informe para la Comisión Nacional de Manglares. UICN, Costa Rica.

Polanía, J. 1993. Mangroves of Costa Rica: p. 129-137. In: L.D. Lacerda. Conservation and sustainable utilization of mangrove forests in Latin America and Africa regions. ITTO/ISME Project PD 114/90 (F), Part I. Okinawa, Japón.

Pool, D.J., S.C. Snedaker \& A.E. Lugo. 1977. Structure of mangrove forests in Florida, Puerto Rico, Mexico and Central America. Biotropica 9: 195-212.

Putz, F.E., G.G. Parker \& R.M. Archibald. 1984. Mechanical abrasion and intercrown spacing. Amer. Midl. Nat. 112: $24-28$.

Ramsar. 1993. Ficha informativa sobre humedales Ramsar. Refugio Nacional de Vida Silvestre Tamarindo. http:// www.wetlands.org/RSDB/default.htm

Ramsar. 1999. Ficha informativa sobre humedales Ramsar. Manglar de Potrero Grande. http://www.wetlands. org/RSDB/default.htm.

Rodgers III, J.C. \& S.P. Horn. 1996. Modern pollen spectra form Costa Rica. Paleogeogr., Paleoclimat., Paleoecol. 124: 53-71.

Silva-Benavides, A. \& R. Bonilla. 2001. Abundancia y morfometría de Anadara tuberculosa y Anadara similis (Mollusca: Bivalvia) en el manglar de Purruja, Golfo Dulce, Costa Rica. Rev. Biol. Trop. 49: 315-320.
Silva, A.M. \& N.N. Carrillo. 2004. El manglar de Purruja, Golfito, Costa Rica: un modelo para su manejo. Rev. Bio. Trop. 52: 195-201.

Solórzano, R., R. de Camino, R. Woodward, J. Tosi, V. Watson, A. Vásquez, C. Villalobos, J. Jiménez, R. Repetto \& W. Cruz. 1991. Accounts overdue: natural resource depreciation in Costa Rica. Tropical Science Center, San José, Costa Rica and World Resources Institute, Washington D.C.

Soto, R. \& L.F. Corrales. 1987. Variación de algunas características foliares de Avicennia germinans (L.) L. (Avicenniaceae) en un gradiente climático y de salinidad. Rev. Biol. Trop. 35: 245-256.

Soto, R. \& J.A. Jiménez. 1982. Análisis fisionómico y estructural del manglar de Puerto Soley, La Cruz, Guanacaste, Costa Rica. Rev. Biol. Trop. 30: 161168 .

Tejada-Rivas, O.L. 2002. Macroalgas asociadas a raíces de mangle: costa Pacífica de Costa Rica. Tesis de Maestría, Universidad de Costa Rica, San Pedro.

Tosi, J.A. 1969. República de Costa Rica, Mapa Ecológico. Escala 1:750 000. Centro Científico Tropical, San José, Costa Rica.

Valiela, I., J.L. Bowen \& J.K. York. 2001. Mangrove forests: one of the world's threatened major tropical environmentes. BioScience 51: 807-815.

Villalobos, C.R., G.A. Cruz \& R.A. Cruz. 1985. Notas sobre la biología de Sphaeroma terebrans Bate, 1866 (Sphaeromatidae: Isopoda) en el manglar de Pochote, Provincia de Puntarenas, Costa Rica. Brenesia 24: 287-295. 